Supporting Information

\title{
Interparticle Charge Transfer Mediated by $\pi-\pi$ Stacking of Aromatic Moieties
}

\author{
Sulolit Pradhan, Debraj Ghosh, Li-Ping Xu, and Shaowei Chen* \\ Department of Chemistry and Biochemistry, University of California, Santa Cruz, \\ California 95064
}

Calculation of Nanoparticle Conductivity. From the I-V slopes, the film resistance (R) was calculated. Then by $49 \mathrm{R}=\rho(\mathrm{L} / \mathrm{S}), \rho$ and hence $\sigma$ was estimated. Here $\mathrm{L}$ is the IDA electrode interfinger gap, $5 \mu \mathrm{m}$; and $\mathrm{S}$ is the film cross section area approximated by (particle physical diameter, core + two ligands) $\times$ (IDA finger length, $3 \mathrm{~mm}$ ). The constant $(49)$ reflects that there are totally 49 junctions which are in parallel within the IDA chip.

Transmission Electron Microscopy (TEM). The particle core diameter was measured with a JEOL 1200 EX Transmission electron microscope (TEM) operated at $80 \mathrm{keV}$. The samples were prepared by casting a drop of the particle solution in dichloromethane onto a 200-mesh carboncoated copper grid. Particle core size was analyzed by using ImageJ software of the obtained micrographs including at least several hundred data points. The average particle core diameters are indicated in the respective figure legend below.

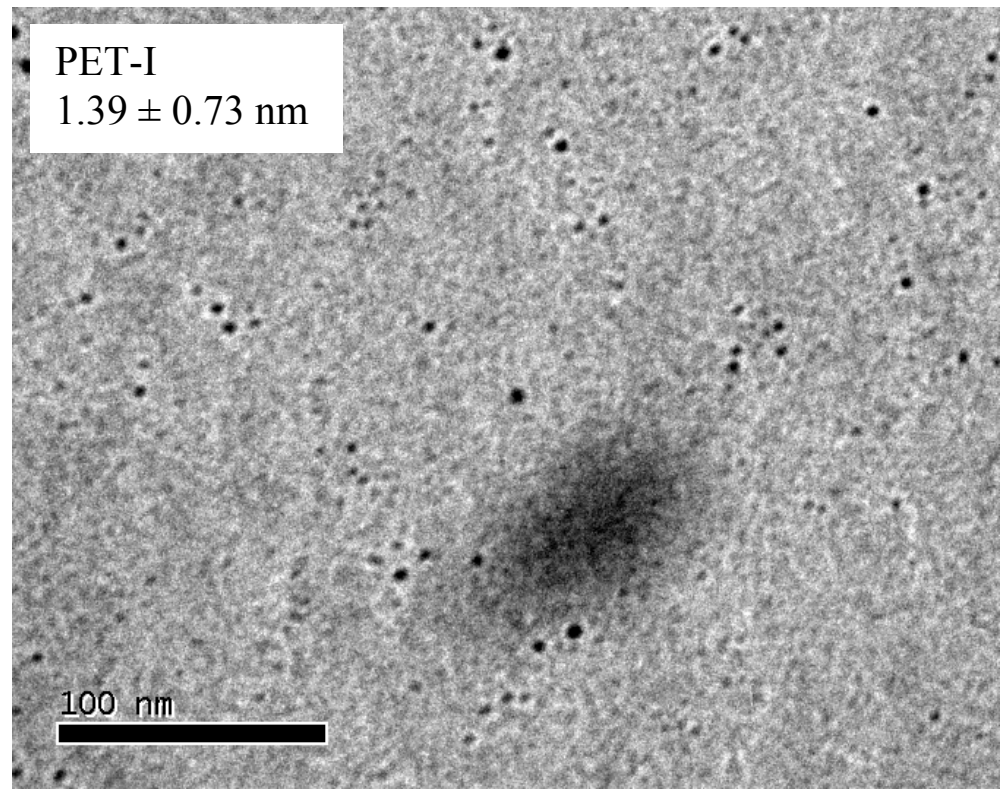



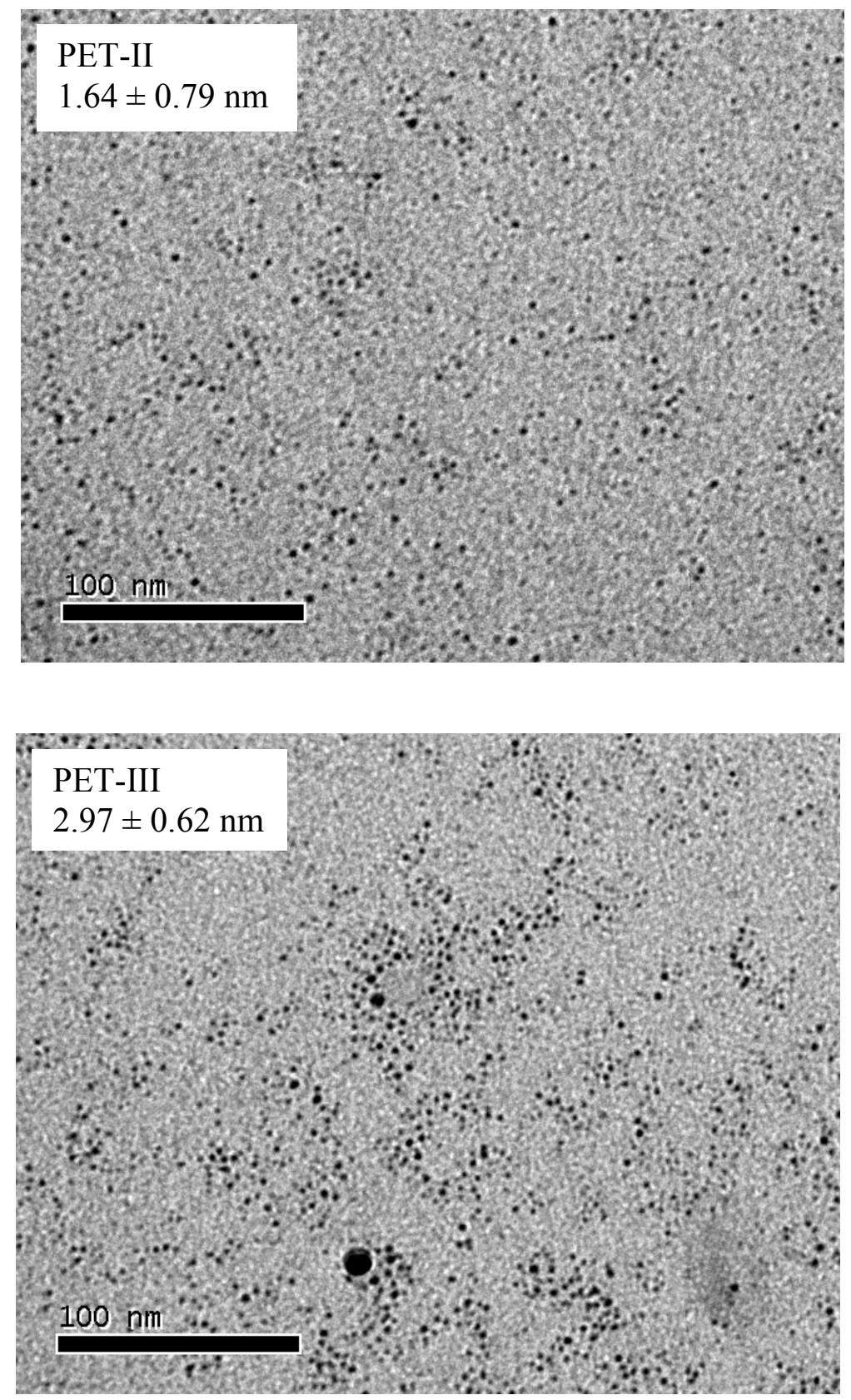

Figure S1. Representative TEM micrographs of PET-Au nanoparticles. Scale bars are all $100 \mathrm{~nm}$. 
Spectroscopy. The particle purity was examined by using ${ }^{1} \mathrm{H}$ NMR spectroscopy (Varian Unity $500 \mathrm{MHz}$ ) with a concentrated solution of particles dissolved in $\mathrm{CDCl}_{3}$. The absence of any sharp features suggests removal of excessive ligands from the particle samples. UV-visible spectroscopic measurements were carried out with a UNICAM ATI UV4 spectrometer. The typical concentration of the particle solution was $0.27 \mu \mathrm{M}$. The spectra are shown in Figure S2. It can be seen that the intensity of the surface plasmon resonance peak (ca. $520 \mathrm{~nm}$ ) decreases in the order of PET-III > PET-II > PET-I, consistent with their core size estimations based on TEM data (Figure S1).

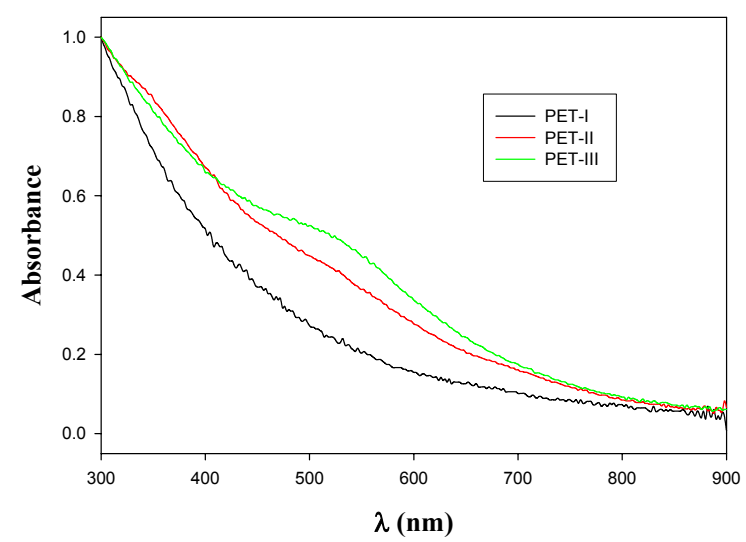

Figure S2. UV-Vis spectra of the three PET-Au nanoparticles. Particle concentration 1 $\mathrm{mg} / \mathrm{mL}$ in toluene. Spectra were normalized to their respective absorbance at $300 \mathrm{~nm}$. 
Langmuir-Blodgett Monolayers of Nanoparticles. The monolayer films of the nanoparticles were prepared by spreading a calculated amount of the particle solutions in toluene onto the water surface of an LB trough (NIMA 611D) by using a Hamilton microliter syringe, and then deposited onto an interdigitated arrays electrode (IDA, consisting of 25 pairs of gold fingers, 5 $\mu \mathrm{m} \times 5 \mu \mathrm{m} \times 3 \mathrm{~mm}$, from ABTECH) by the LB method at varied interparticle separations. The dipper speed was controlled at $1 \mathrm{~mm} / \mathrm{min}$.

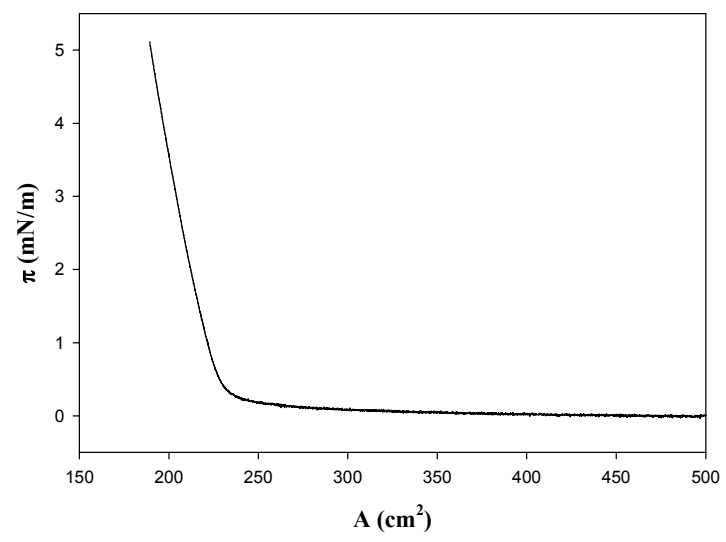

Figure S3. Representative Langmuir isotherm of PET-Au nanoparticles (PET-III). Particle concentration $1 \mathrm{mg} / \mathrm{mL}$ in toluene; amount spread $200 \mu \mathrm{L}$. Compression speed $10 \mathrm{~cm}^{2} / \mathrm{min}$.

Table S1 Selected comparison of the interparticle distances $(l)$ calculated from the Langmuir isotherm and from TEM measurements for the PET-III nanoparticle monolayers

\begin{tabular}{|c|c|c|}
\hline $\begin{array}{c}\text { Langmuir Distance } \\
(\mathbf{n m})\end{array}$ & $\begin{array}{c}\text { TEM Distance } \\
(\mathbf{n m})\end{array}$ & $\begin{array}{c}\text { TEM Image } \\
\text { (scale bar }=\mathbf{8 . 4 6} \mathbf{n m} \text { ) }\end{array}$ \\
\hline 0.68 & $0.69 \pm 0.12$ & $(\mathrm{~A})$ \\
\hline 0.80 & $0.77 \pm 0.15$ & $(\mathrm{~B})$ \\
\hline 0.90 & $0.86 \pm 0.22$ & (C) \\
\hline
\end{tabular}

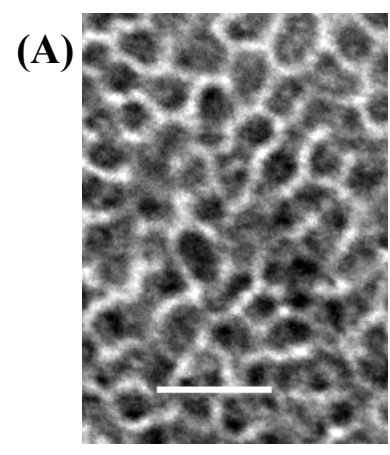

(B)

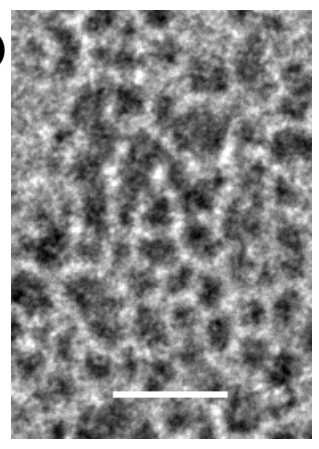

(C)

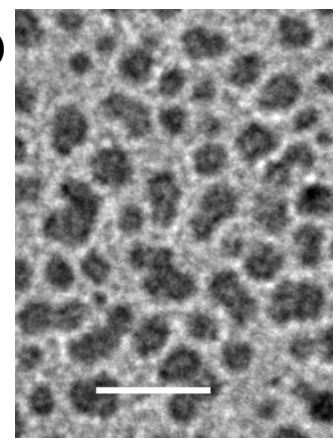


Scanning Tunneling Microscopy (STM). To verify the structural integrity of the nanoparticle LB monolayer films, nanoparticle monolayers were also deposited onto a $\mathrm{Au}(111)$ substrate surface for STM studies (PicoLE SPM from Molecular Imaging) under exactly the same conditions as those for IDA deposition. In STM measurements, atomically flat Au(111) substrate was used which was coated a prior with a decanethiol self-assembled monolayer to render the surface hydrophobic and enhance the interactions with the particles. A mechanically cut Pt/Ir tip was used in the STM measurements.

Figure S4 depicts the STM images of four PET-III nanoparticle monolayers at varied interparticle separations. The insets show the histograms of the edge-edge distance as estimated from the corresponding STM topographs. Table S1 compares the distances from the STM measurements with those based on the deposition areas (Langmuir isotherm). It can be seen that they show very good agreement, though some deviation can be found at very large or very small interparticle separation.

Figure S5 shows similar measurements with the PET-I and PET-II nanoparticle monolayers. Both were deposited at $l=0.90 \mathrm{~nm}$ (from Langmuir isotherms). The distance as estimated from the STM measurement is somewhat larger, at 1.02 and $0.97 \mathrm{~nm}$ respectively.

Table S2 Interparticle distances $(l)$ calculated from the Langmuir isotherm and from STM measurements for the PET-III nanoparticle monolayers

\begin{tabular}{|c|c|}
\hline $\begin{array}{c}\text { Langmuir Distance } \\
(\mathbf{n m})\end{array}$ & $\begin{array}{c}\text { STM Distance } \\
\text { (nm) }\end{array}$ \\
\hline 0.69 & $0.58 \pm 0.03$ \\
\hline 0.90 & $0.96 \pm 0.07$ \\
\hline 1.11 & $1.12 \pm 0.23$ \\
\hline 1.20 & $1.84 \pm 0.18$ \\
\hline
\end{tabular}



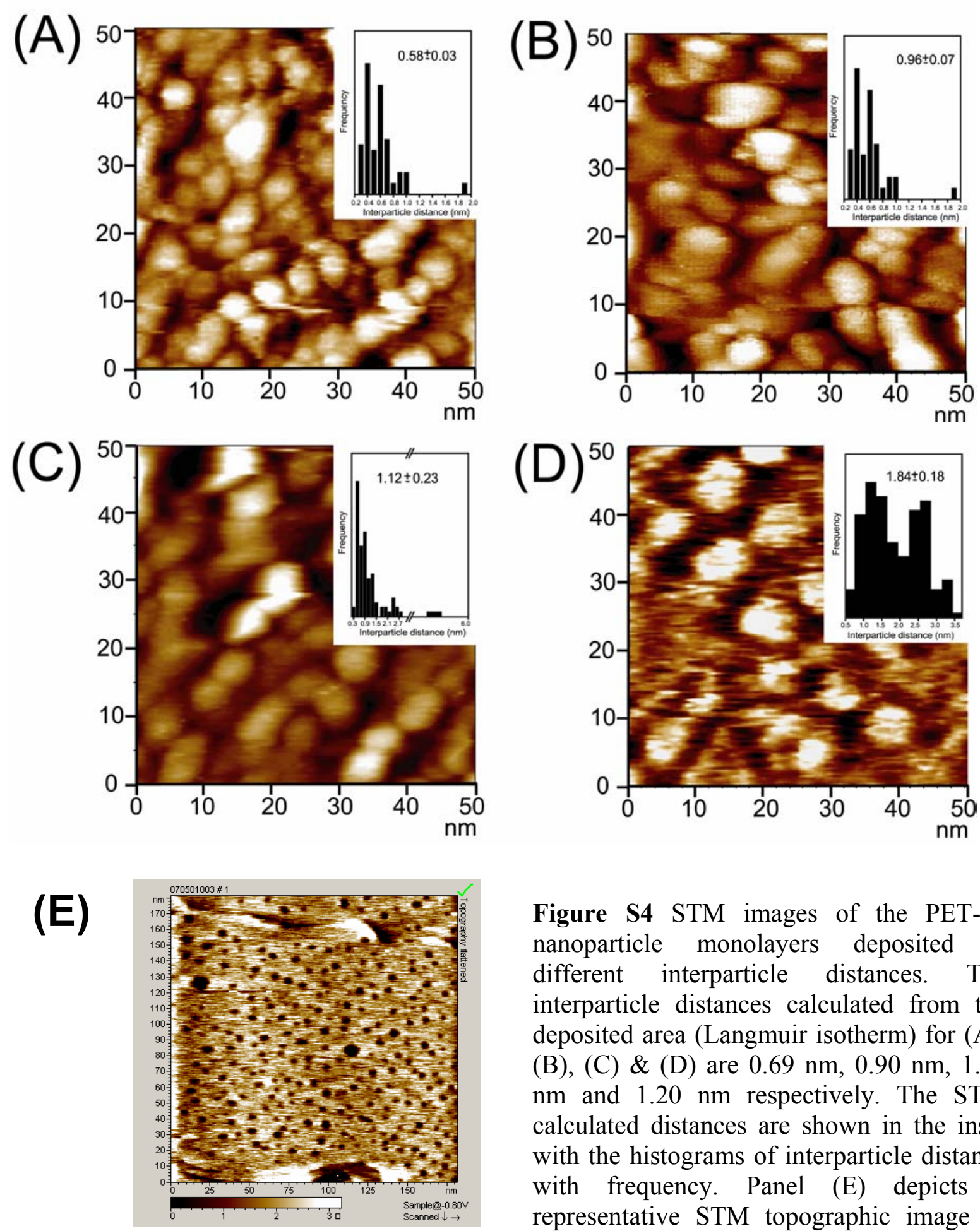

Figure S4 STM images of the PET-III nanoparticle monolayers deposited at different interparticle distances. The interparticle distances calculated from the deposited area (Langmuir isotherm) for (A), (B), (C) \& (D) are $0.69 \mathrm{~nm}, 0.90 \mathrm{~nm}, 1.11$ $\mathrm{nm}$ and $1.20 \mathrm{~nm}$ respectively. The STM calculated distances are shown in the inset with the histograms of interparticle distance with frequency. Panel (E) depicts a representative STM topographic image of the $\mathrm{Au}(111)$ coated with a decanethiol selfassembled monolayer prior to particle deposition. 

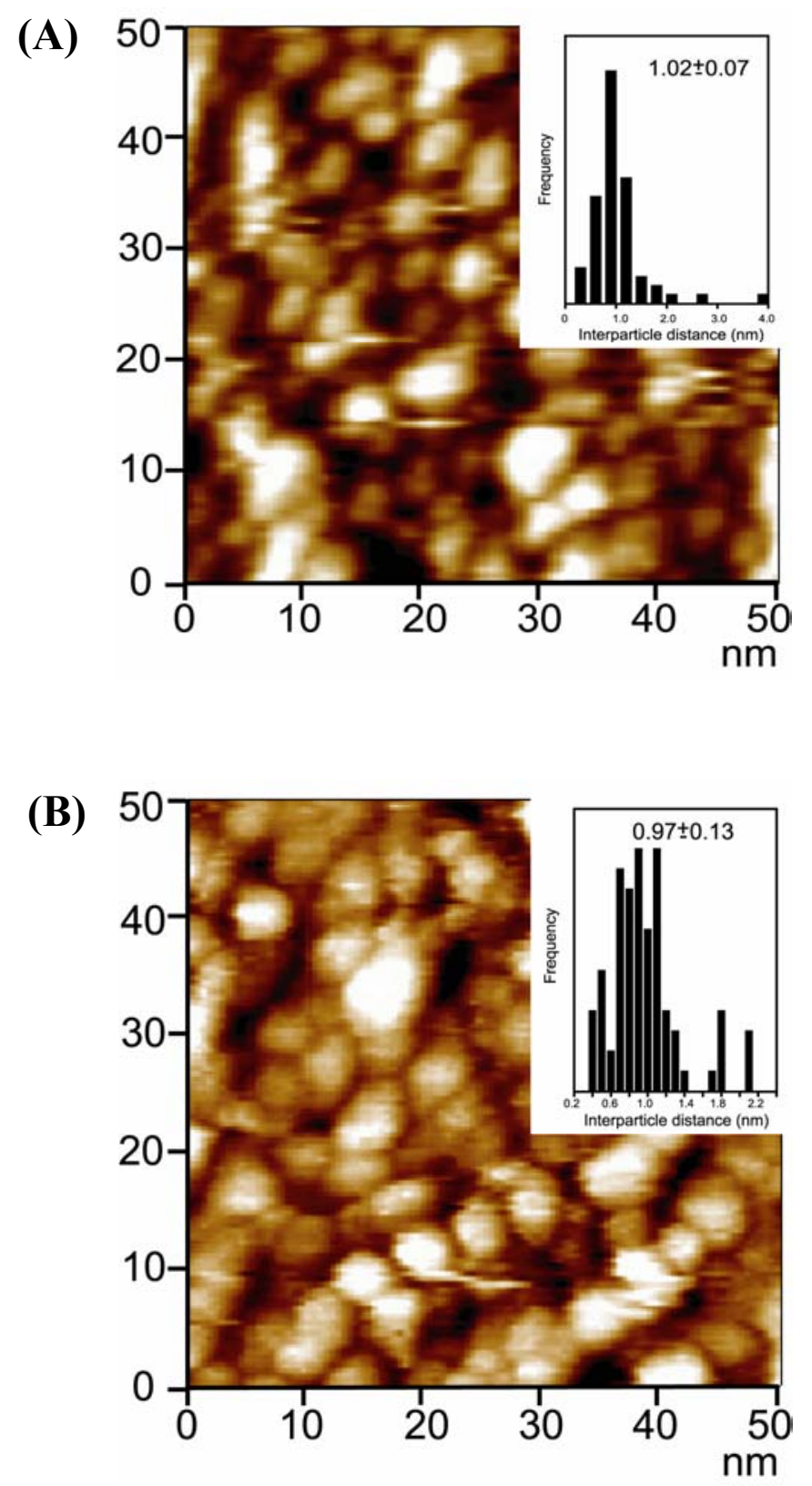

Figure S5 STM images of PET-I (A) and PET-II (B) nanoparticle monolayers at an interparticle distance of $0.90 \mathrm{~nm}$ as estimated from Langmuir isotherm based on the deposition area. The STM calculated distances are shown in the inset with the histograms of interparticle distance with frequency. 
Electrochemistry. Electrochemical measurements were carried out with an EG\&G PARC 283 Potentiostat/Galvanostat in vacuo with a cryostat from Janis Research and at different temperatures (Lakeshore 331 Temperature Controller).

Figure S6 shows a representative plot of the monolayer conductivity as a function of temperature. It can be seen that (i) the conductivity of the particle monolayers increases with increasing temperature; and (ii) at temperature $\geq 280 \mathrm{~K}$, the particle conductivity exhibits a linear increase with $\mathrm{T}^{-1}$ with an activation energy much larger than that found at lower temperature (assuming similar Arrhenius behaviors). Such dual dependence of conductivity on temperature has also been observed previously with LB monolayers of alkanethiolate-protected nanoparticles and ascribed to the variation of interparticle charge transfer mechanism with temperature (ref. 8).

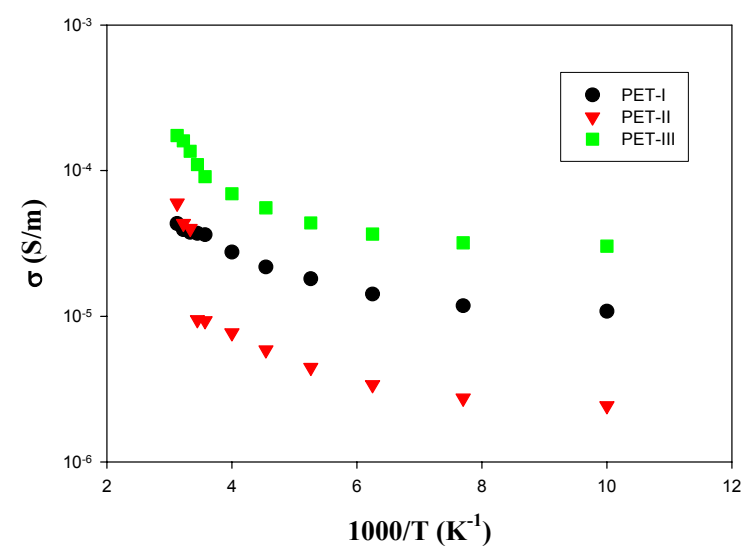

Figurer S6. Representative Arrhenius plots of the LB monolayers of PET-Au nanoparticles. Data were calculated from the respective I-V profiles shown in Figure 1 insets. 
Nanoparticle Dropcast Thick Films. In these measurements, a calculated amount of a concentrated solution of the nanoparticles in toluene was dropcast onto the same IDA electrode (totally $25 \mu \mathrm{g}$ of particles) by using a Hamilton microliter syringe. The films were then examined under an optical microscope to make sure that the entire IDA fingers were buried under the films. The particle films were then dried with a gentle stream of ultra-high purity nitrogen before being introduced into the cryostat for electrochemical measurements.

Figure S7 shows the corresponding I-V profiles of the three gold nanoparticles, along with the temperature dependence of the film conductivity (Figure S8). It can be seen that the particle conductivity increases and activation energy decreases with increasing core size. Activation energies for the dropcast films evaluated from the plot (Figure S8) were $93.5 \mathrm{meV}$ (PET-I); 16.8 $\mathrm{meV}$ (PET-II), and $10.4 \mathrm{meV}$ (PET-III), which are comparable to those for the LB monolayers, $66.6 \mathrm{meV}$ (PET-I), $20.5 \mathrm{meV}$ (PET-II) and $3.5 \mathrm{meV}$ (PET-III). Note that Murray et al (ref. 3) reported that the activation energy for arenethiolate-capped gold nanoparticle solid films was in the range of 40 to $80 \mathrm{meV}$, although one should compare the data with caution as the particle core size and dispersity are different in these two studies.

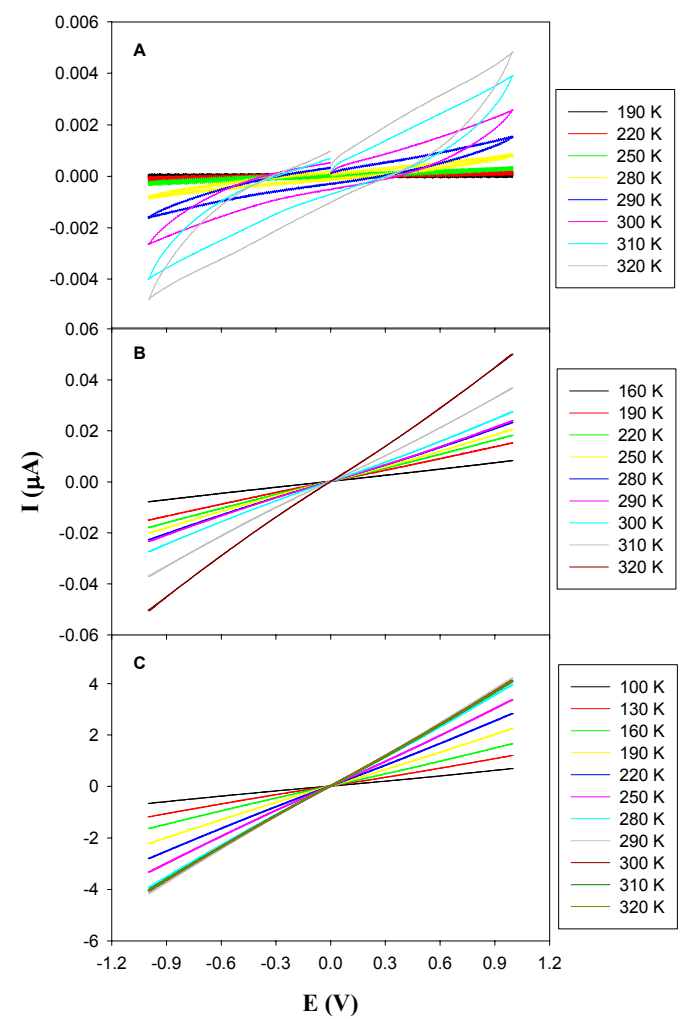

Figure S7. I-V profiles of dropcast thick films of the three PET-Au nanoparticles at varied temperatures: (A) PET-I, (B) PET-II, and (C) PET-III.

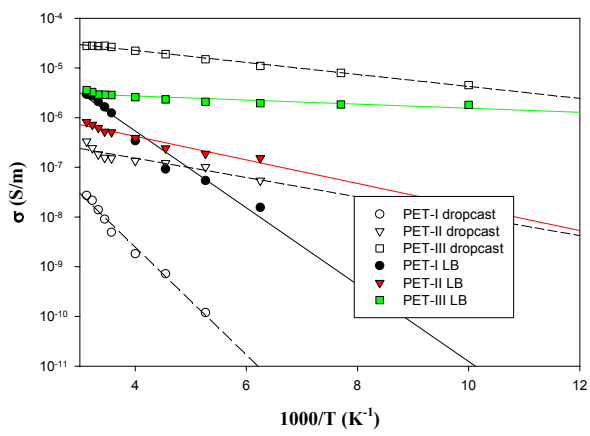

Figure S8. Arrhenius plots of dropcast thick films and LB monolayers (at $l=1.36 \mathrm{~nm}$ ) of PET-Au nanoparticles. Symbols are experimental data calculated from Figure S7 and Figure 1 respectively. Lines are linear regressions. 